Int. J. Electrochem. Sci., 13 (2018) 3475 - 3485

\title{
Comparing the Support Effects of Graphene Nanosheets (GNs) and N-doped GNs with Respect to Anti-Poisoning Performance of Pt Catalysts
}

\author{
Yujie Yang ${ }^{1}$, Jianshe Wang ${ }^{1, *}$, Changhai Liu ${ }^{2, *}$, Niancai Cheng ${ }^{3}$, Xueliang Sun ${ }^{4}$ \\ ${ }^{1}$ School of Chemical Engineering and Energy, Zhengzhou University, Zhengzhou, Henan, 450000, P. \\ R. China \\ ${ }^{2}$ School of Materials Science \& Engineering, Jiangsu Collaborative Innovation Center of Photovoltaic \\ Science and Engineering, Changzhou University, Changzhou, Jiangsu 213164, P.R. China \\ ${ }^{3}$ School of Materials Science \& Engineering, Fuzhou University, Fuzhou, Fujian, 350116, P. R. China \\ ${ }^{4}$ Department of Mechanical and Materials Engineering, The University of Western Ontario, ON, N6A \\ 5B9, Canada \\ *E-mail: wangjs07@zzu.edu.cn, liuch@cczu.edu.cn.
}

doi: $10.20964 / 2018.04 .18$

Received: 9 January 2018 / Accepted: 5 February 2018 / Published: 6 March 2018

It is of great significance to precisely evaluate the anti-poisoning performance (APP) of anode catalysts for development of direct liquids fuel cells. By controlloing the similarity of Pt deposited on graphene nanosheets (GNs) and N-dopped GNs (NGNs), Pt/GNs and Pt/NGNs were prepared as model catalysts for APP evaluation. Cyclic voltammograms (CVs) of Pt/GNs and Pt/NGNs for formate and methanol oxidation showed that the APP differences of two catalysts were hardly distinguished only from CVs because the activities of the two catalysts were similar. By further analyzing the i-t data, Pt/NGNs was proved to be evidently superior to Pt/GNs in terms of APP, verifying the necessity of APP analysis for comprehensive catalysts evaluation. Furthermore, by analyzing Raman spectra, $\mathrm{CO}$ stripping and X-ray photoelectron spectroscopy, stronger interaction between Pt and NGNs was shown to produce weaker adsorption of poisoning species on NGNs-supported Pt, which is benificial for enhancing APP of Pt/NGNs. We believe this understanding can shed light on future work toward rational supports engineering for APP improvement.

Keywords: Anti-poisoning performance; Pt catalysts; graphene; i-t measurement; formate oxidation

\section{$\underline{\text { FULL TEXT }}$}

(C) 2018 The Authors. Published by ESG (www.electrochemsci.org). This article is an open access article distributed under the terms and conditions of the Creative Commons Attribution license (http://creativecommons.org/licenses/by/4.0/). 